Supporting Information

\title{
Efficient water self-diffusion in diphenylalanine peptide nanotubes
}

Pavel S. Zelenovskiy, Eddy M. Domingues, Vladislav Slabov, Svitlana Kopyl, Valery L. Ugolkov, Filipe M. L. Figueiredo, Andrei L. Kholkin

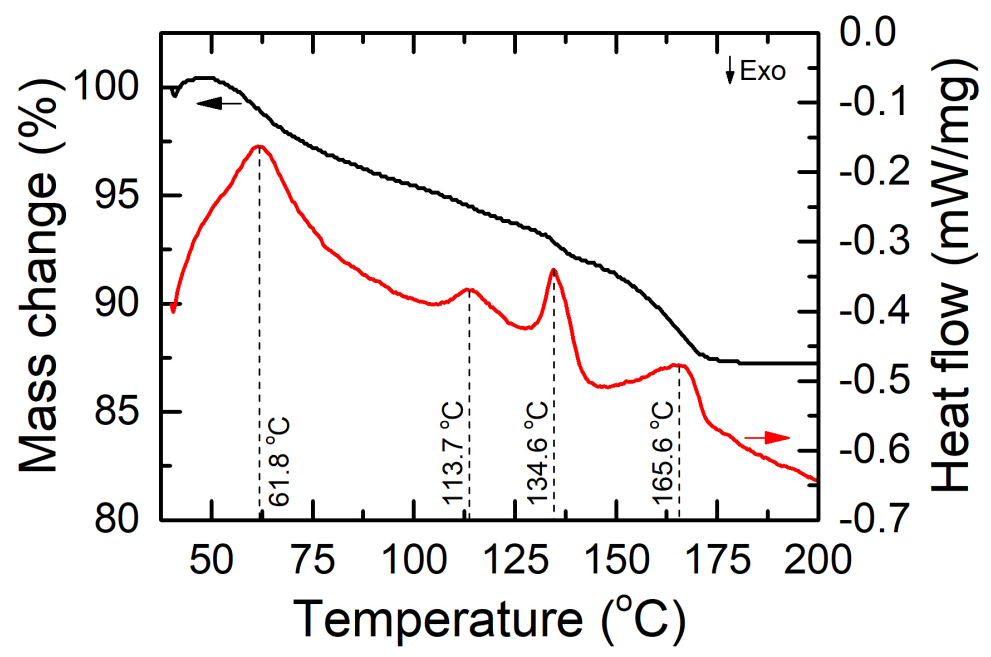

Figure S1. DSC (red) and TGA (black) curves of FF NTs.
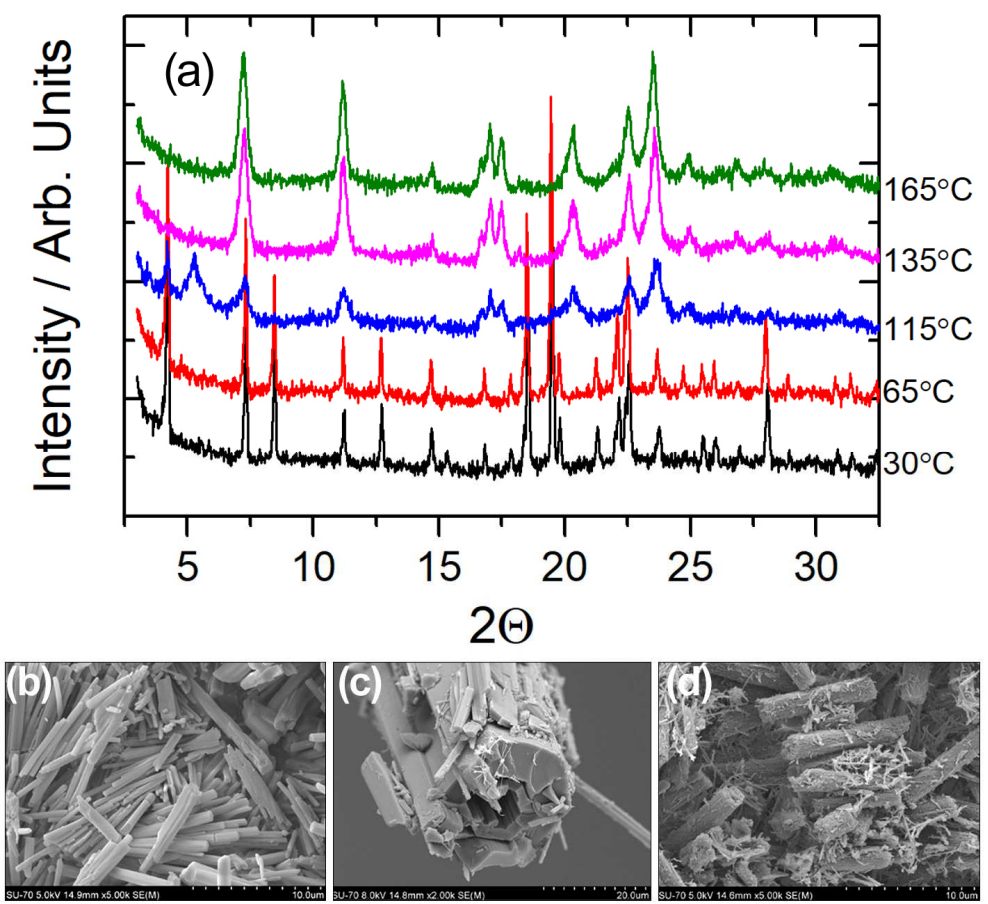

Figure S2. (a) XRD patterns of FF NTs at temperatures corresponding to peaks on the DSC curve, denoting significant structural changes at $115^{\circ} \mathrm{C}$ and higher temperatures. (b-d) SEM micrographs of the FF microtubes morphology at (b) initial state and after the dehydration at (c) $65^{\circ} \mathrm{C}$ and (d) $135^{\circ} \mathrm{C}$. 


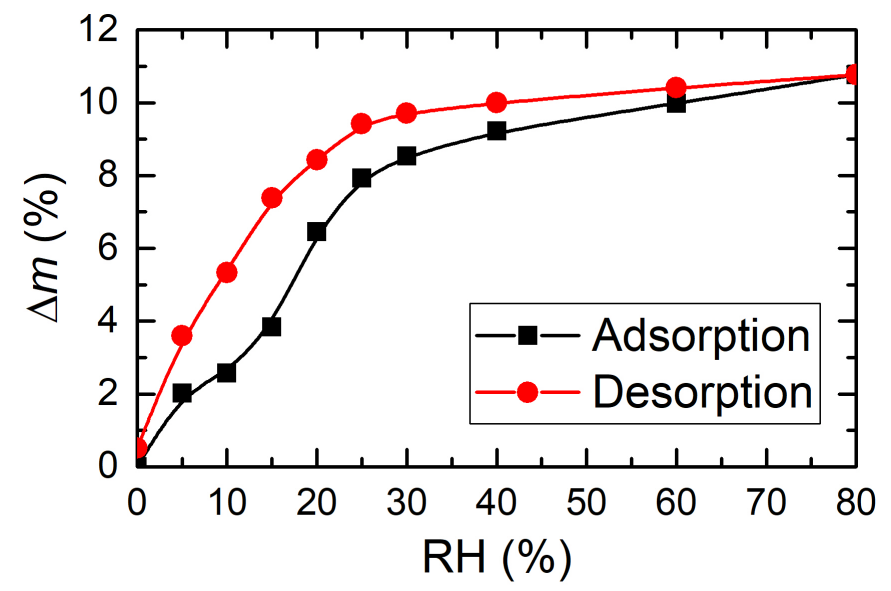

Figure S3. Water adsorption-desorption isotherms for FF NTs measured at $50^{\circ} \mathrm{C}$ in extended RH range.
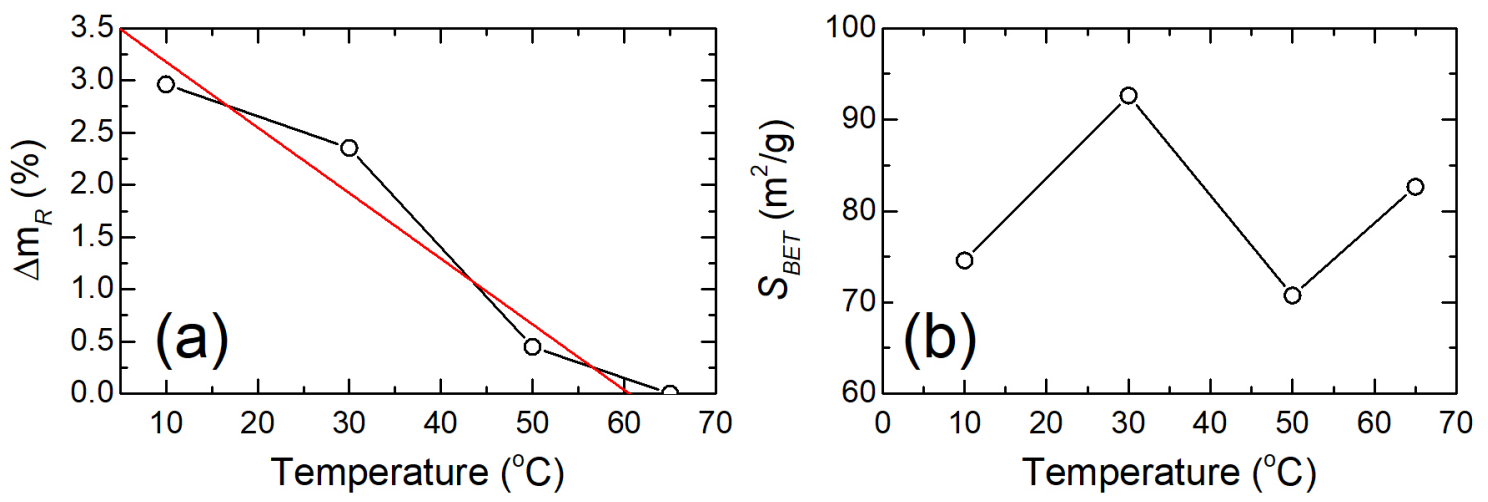

Figure S4. Temperature dependence of the (a) residual mass of adsorbed water at $\mathrm{RH}=0 \%$ after desorption, and of the (b) specific surface area, $S_{B E T}$. 


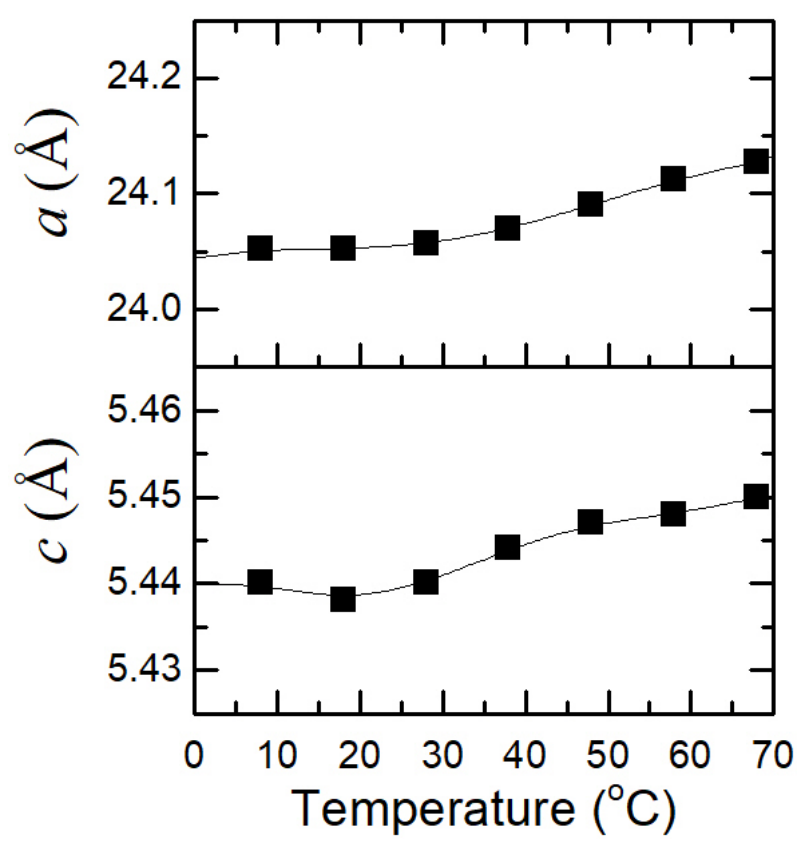

Figure S5. Temperature variations of the cell parameters of FF nanotubes.

\section{Estimation of the correction coefficient to the BET surface area}

Crystallographic data show that water molecules in the nanochannels of FF NTs are arranged into a helix, where two water molecules are bounded with one FF molecule (Figure S6a). The fraction of the surface area occupied by bound water molecules in the FF NTs can thus be obtained by considering the area of the helical stripe covering the cylinder inner surface (Figure S6b). The width of the stripe, $w$, equals the diameter of the water molecule $(w=0.265 \mathrm{~nm})$, the step of the helix, $C$, corresponds to the parameter $c$ of the NT crystallographic unit cell $(C=0.55 \mathrm{~nm})$, and the cylinder diameter, $d$, corresponds to the internal diameter of the FF NT $(d=1 \mathrm{~nm})$. 
(a)

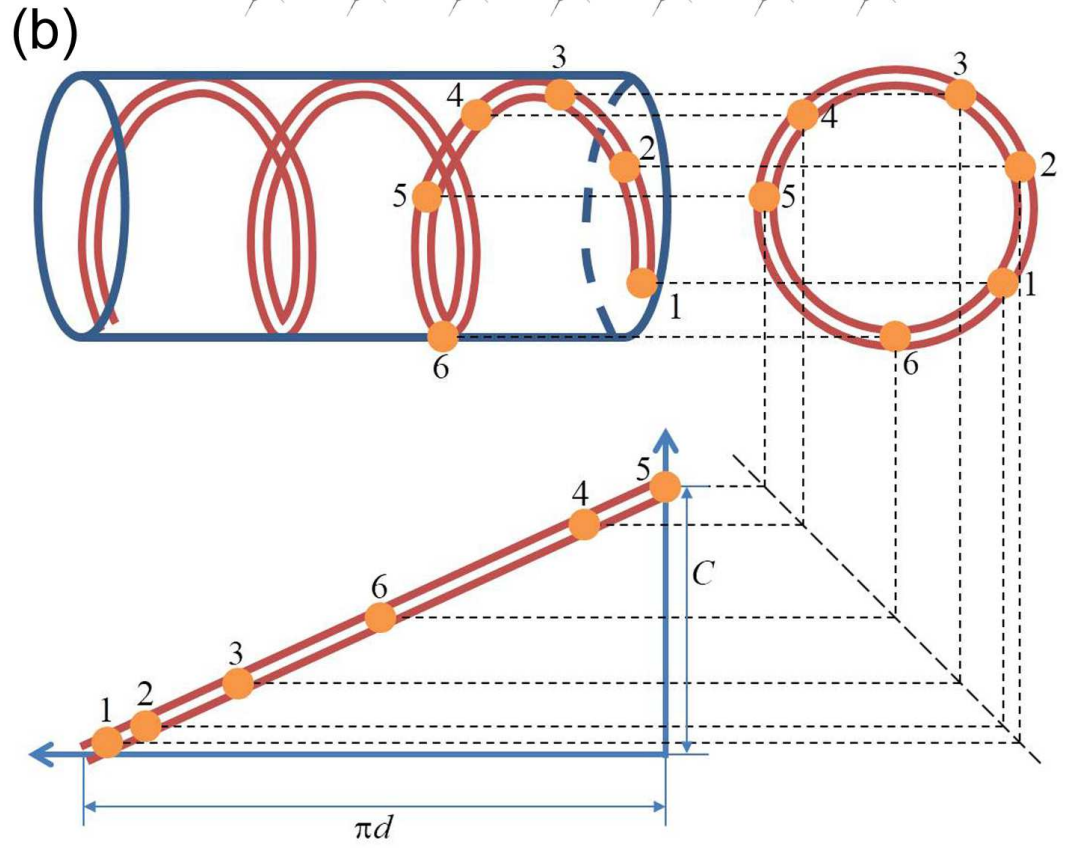

Figure S6. (a) Cross section of FF NT with water molecules inside,

and (b) scheme for the estimation of the surface area occupied by water molecules.

The area of the stripe, $S_{W}$, representing the BET surface area obtained from water adsorption measurements, is equal to $S_{W}=L \cdot w$, were $L=\sqrt{\pi^{2} d^{2}+C^{2}}$ is the length of the stripe found from the stripe evolvent (Figure S6b). The full area of the cylinder corresponding to the geometrical surface area is $S_{C}=\pi d C$. Therefore, the $S_{W} / S_{G}$ ratio is given by:

$$
\frac{S_{W}}{S_{C}} \equiv \frac{S_{B E T}}{S_{G}}=\frac{w}{d} \sqrt{\frac{d^{2}}{C^{2}}+\frac{1}{\pi^{2}}} \approx 0.493 .
$$


Since the inner diameter of the nanochannel is comparable with the size of the water molecule a one-dimensional scenario can be considered. In the simplest approach, water vapor diffusion through a NT of $2 \times x_{0}$ length with impermeable walls (Figure S7a) can be reduced to the problem of filling of a half-length tube with one open end (Figure S7b), with the equivalent and symmetric conditions at both ends of the NT.

Temporal variation of water concentration over the model NT is described by the steady-state diffusion equation:

$$
\frac{\partial C(x, t)}{\partial t}=D \frac{\partial^{2} C(x, t)}{\partial x^{2}}
$$

where $C(x, t)$ is the water concentration, and $D$ is the diffusion coefficient. This equation requires the determination of initial and boundary conditions. We suppose that the NT is empty at $t=0$, and the water concentration outside the tube (at $x=x_{0}$ ) is constant. Due to the symmetry of the problem, the other end of the NT (at $x=0)$ is considered impermeable. All these conditions can be written in the form:

$$
\begin{gathered}
C(x, 0)=0 . \\
C\left(x_{0}, t\right)=C_{0} . \\
\left.\frac{\partial C(x, t)}{\partial x}\right|_{x=0}=0 .
\end{gathered}
$$

The general solution of Eq. (1) with initial and boundary conditions (2)-(4) is [S2]:

$$
C(x, t)=C_{0}\left[1-\sum_{n=0}^{\infty}(-1)^{n} \frac{4}{(2 n+1) \pi} \cos \left(\pi \frac{2 n+1}{2} \frac{x}{x_{0}}\right) \exp \left(-\frac{D}{x_{0}^{2}} \frac{(2 n+1)^{2}}{4} \pi^{2} t\right)\right] \text {. }
$$




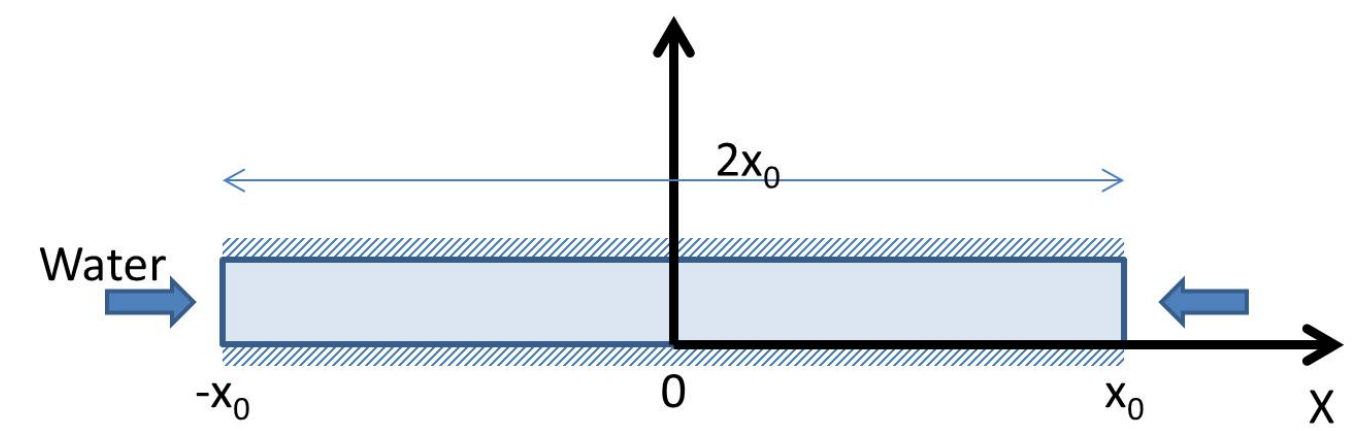

(a)

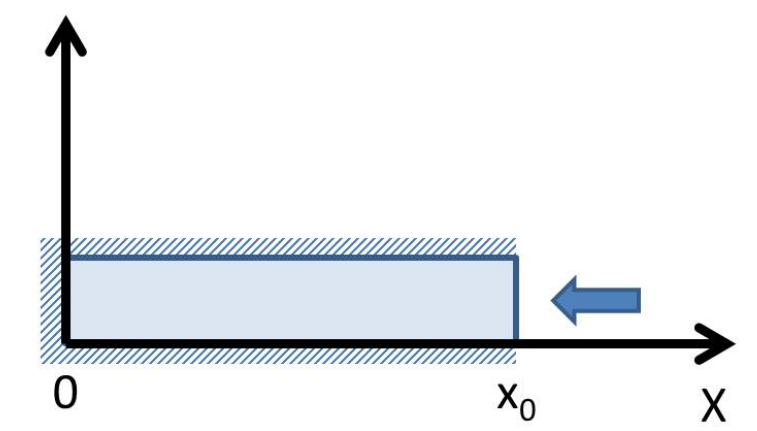

(b)

Figure S7. (a) Full and (b) reduced models of NT for study the water diffusion kinetics through FF nanochannels. Blue arrows show the direction of water vapor propagation

Integration of Eq. (5) over the length of the model NT gives the time variation of the mass $M$ of the water vapor in the nanochannel:

$$
M(t)=\int_{0}^{x_{0}} C(x, t) d x=C_{0} x_{0}\left[1-\sum_{n=0}^{\infty} \frac{8}{(2 n+1)^{2} \pi^{2}} \exp \left(-\frac{D}{x_{0}^{2}} \frac{(2 n+1)^{2}}{4} \pi^{2} t\right)\right] .
$$

Figure S7 further shows that only half of the tube is considered in the model. Therefore a factor of 2 should be additionally written in Eq. (6) in order to obtain the total mass of the adsorbed water. The speed of water diffusion (adsorption) can be found as the time derivative of Eq. (6):

$$
\frac{\partial M}{\partial t}=4 C_{0} \frac{D}{x_{0}} \sum_{n=0}^{\infty} \exp \left(-\frac{D}{x_{0}^{2}} \frac{(2 n+1)^{2}}{4} \pi^{2} t\right)
$$


This expression includes an infinite sum, which for practical purposes can be restricted to the first three terms:

$$
\frac{\partial M}{\partial t}(t)=4 C_{0} \frac{D}{x_{0}}\left[\exp \left(-\frac{D}{x_{0}^{2}} \frac{\pi^{2}}{4} t\right)+\exp \left(-\frac{D}{x_{0}^{2}} \frac{9 \pi^{2}}{4} t\right)+\exp \left(-\frac{D}{x_{0}^{2}} \frac{25 \pi^{2}}{4} t\right)\right]
$$

This equation can be used for the determination of the diffusion coefficient $D$ from adsorption-desorption measurements. We have shown recently, on basis of large statistical data, that the mode length (the most probable length) of FF MTs grown under ambient conditions is $490 \mu \mathrm{m}$ [S1], and thus $x_{0}=245 \mu \mathrm{m}$. Typical experimental data of the experimental temporal variation of the mass of adsorbed water at $30^{\circ} \mathrm{C}$ and $\mathrm{RH}=2 \%$ is presented in Figure S8a, and the corresponding time derivative - in Figure S8b. The fitting of the decayed part of $\partial M / \partial t$ data by Eq. (8) allowed the determination of the value of $D=(0.44 \pm 0.01) \times 10^{-11} \mathrm{~m}^{2} \mathrm{~s}^{-1}$. At room temperature and $\mathrm{RH}=38 \%$ the $D$ reaches a maximum of $1.3 \times 10^{-10} \mathrm{~m}^{2} \mathrm{~s}^{-1}$. The same rationale was applied to all segments of the sorption isotherms to systematically obtain $D$ values as a function of $\mathrm{RH}$ and temperature.

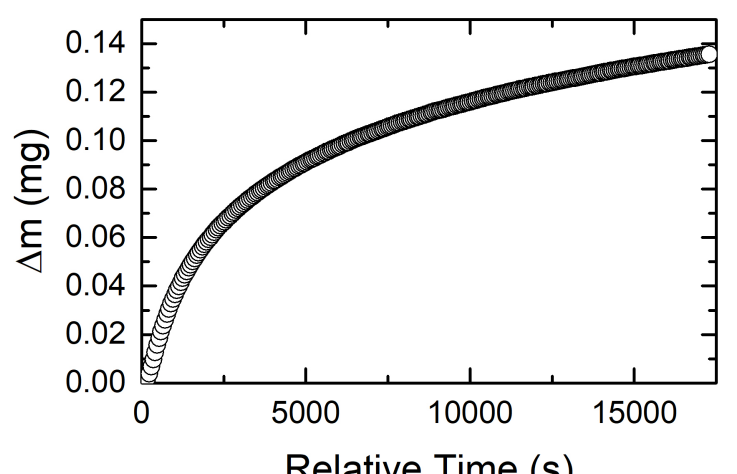

(a)

Relative Time (s)

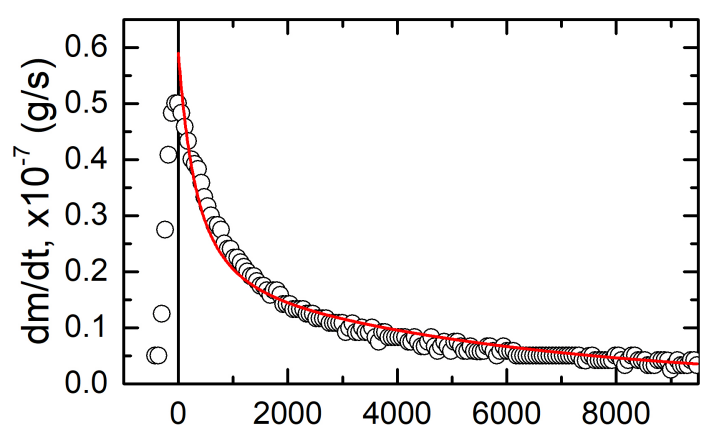

(b)

Relative Time (s)

Figure S8. Temporal variation of (a) mass of adsorbed water and (b) speed of mass variation during the water adsorption at $30{ }^{\circ} \mathrm{C}$ and $\mathrm{RH}=2 \%$. The red line represents fitting by Eq. (8). 
Determination of the number of adsorbed water molecules inside the nanochannels

The total mass $M$ of the sample at certain RH consists of the mass of FF molecules, $M_{F F}$, and the mass of the water in the sample, $M_{W}$. The $M_{F F}$ can be expressed as $M_{F F}=m_{F F} \cdot N_{F F}$, where $m_{F F}$ is the mass of one FF molecule, and $N_{F F}$ is the number of FF molecules in the sample. In turn, $M_{W}$ depends on the number of water molecules in the initial, dry state, $N_{W}(0)$, and the number of molecules adsorbed by the sample at current RH, $N_{W}$. Therefore, the total mass of the sample is:

$$
M=M_{F F}+M_{W}=m_{F F} \cdot N_{F F}+m_{W} \cdot N_{W}(0)+m_{W} \cdot N_{W}=M_{0}+m_{W} \cdot N_{W},
$$

where $M_{0}=m_{F F} \cdot N_{F F}+m_{W} \cdot N_{W}(0)$ is the initial mass of the sample and $m_{W}$ is the mass of one water molecule. Dividing this equation by $M_{0}$ one obtains the following expression:

$$
\frac{M}{M_{0}}=1+\frac{m_{W} \cdot N_{W}}{m_{F F} \cdot N_{F F}+m_{W} \cdot N_{W}(0)}
$$

The fraction in the right part of the equation can be simplified by dividing the numerator and denominator by $m_{W} \cdot N_{F F}$. Therefore:

$$
\frac{M}{M_{0}}=1+\frac{N_{W} / N_{F F}}{m_{F F} / m_{W}+N_{W}(0) / N_{F F}},
$$

whence it follows that the average number of adsorbed water per one FF molecule is:

$$
\frac{N_{W}}{N_{F F}}=\left(\frac{M}{M_{0}}-1\right)\left(\frac{m_{F F}}{m_{W}}+\frac{N_{W}(0)}{N_{F F}}\right) .
$$

The ratio of masses $m_{F F} / m_{W}=312 / 18=17.3$. In accordance with crystallographic data obtained by single crystal X-ray scattering [S1] and considering the complete filling of the first hydration layer, one can suppose that $N_{W}(0) / N_{F F}=2$. Thus, the correlation between the mass of adsorbed water and the average number of adsorbed water molecules inside the NT unit cell can be obtained, of which an example is given in Figure S9. 


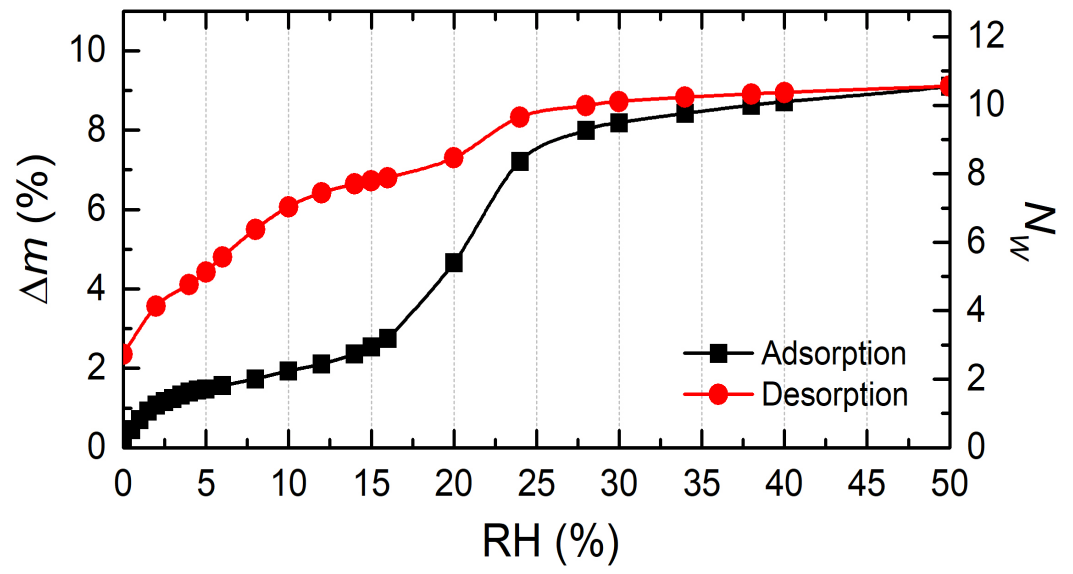

Figure S9. Typical water adsorption-desorption isotherms for FF NTs at $30^{\circ} \mathrm{C}$, where $N_{W}$ is the average number of adsorbed water molecules inside the NT unit cell calculated from the mass of adsorbed water.

Table S1. Parameters of linear fitting of $\log (D)$ vs. $N_{W}$

\begin{tabular}{|c|c|c|c|c|c|c|}
\hline \multirow{2}{*}{ Temperature, ${ }^{\circ} \mathrm{C}$} & \multicolumn{3}{|c|}{ Adsorption } & \multicolumn{3}{c|}{ Desorption } \\
\cline { 2 - 7 } & Slope 1 & $\mathrm{N}_{\mathrm{W}}{ }^{\text {th }}$ & Slope 2 & Slope 1 & $\mathrm{N}_{\mathrm{W}}{ }^{\text {th }}$ & Slope 2 \\
\hline 10 & $-0.43 \pm 0.12$ & $5.52 \pm 0.43$ & $0.89 \pm 0.13$ & - & - & $0.41 \pm 0.04$ \\
\hline 30 & $-0.47 \pm 0.07$ & $5.40 \pm 0.38$ & $0.65 \pm 0.08$ & - & - & $0.32 \pm 0.03$ \\
\hline 50 & $-0.21 \pm 0.08$ & $5.35 \pm 0.63$ & $0.29 \pm 0.07$ & - & - & $0.23 \pm 0.02$ \\
\hline 65 & $-0.30 \pm 0.06$ & $3.49 \pm 0.35$ & $0.17 \pm 0.04$ & $-1.34 \pm 0.28$ & $3.75 \pm 0.17$ & $0.25 \pm 0.02$ \\
\hline
\end{tabular}



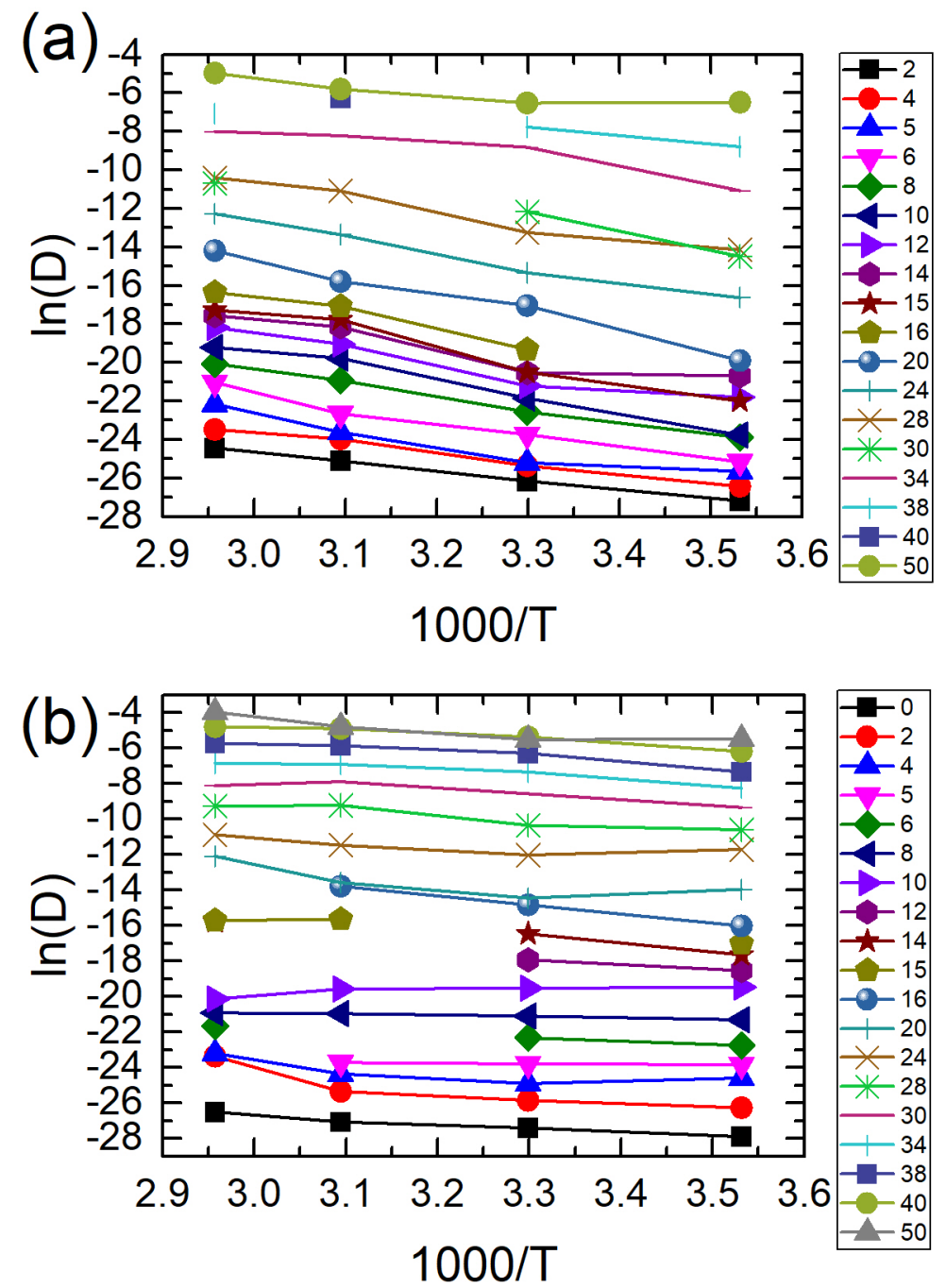

Figure S10. Arrhenius plots for water self-diffusion coefficients estimated from (a) adsorption and (b) desorption isotherms under variable RH. A stack representation with shift 1 is retained for the sake of clarity.

\section{Supplementary references}

[S1] Zelenovskiy, P. S.; Nuraeva, A. S.; Kopyl, S.; Arkhipov, S. G.; Vasilev, S. G.; Bystrov, V. S.; Gruzdev, D. A.; Waliczek, M.; Svitlyk, V.; Shur, V. Ya.; Mafra, L.; Kholkin, A. L. Chirality-Dependent Growth of Self-Assembled Diphenylalanine Microtubes. Cryst. Growth Des. 2019, 19, 6414-6421.

[S2] J. Crank, The Mathematics of Diffusion, Clarendon Press, Oxford, UK 1975. 